\title{
Dynamics of the deep red Fe3+ photoluminescence emission in feldspar
}

\author{
Prasad, Amit Kumar; Jain, Mayank
}

Published in:

Journal of Luminescence

Link to article, DOI:

10.1016/j.jlumin.2017.11.051

Publication date:

2018

Document Version

Peer reviewed version

Link back to DTU Orbit

Citation (APA):

Prasad, A. K., \& Jain, M. (2018). Dynamics of the deep red $\mathrm{Fe}^{3+}$ photoluminescence emission in feldspar. Journal of Luminescence, 196, 462-469. https://doi.org/10.1016/j.jlumin.2017.11.051

\section{General rights}

Copyright and moral rights for the publications made accessible in the public portal are retained by the authors and/or other copyright owners and it is a condition of accessing publications that users recognise and abide by the legal requirements associated with these rights.

- Users may download and print one copy of any publication from the public portal for the purpose of private study or research.

- You may not further distribute the material or use it for any profit-making activity or commercial gain

- You may freely distribute the URL identifying the publication in the public portal

If you believe that this document breaches copyright please contact us providing details, and we will remove access to the work immediately and investigate your claim. 


\section{Author's Accepted Manuscript}

Dynamics of the deep red $\mathrm{Fe}^{3+}$ photoluminescence emission in feldspar

Amit Kumar Prasad, Mayank Jain

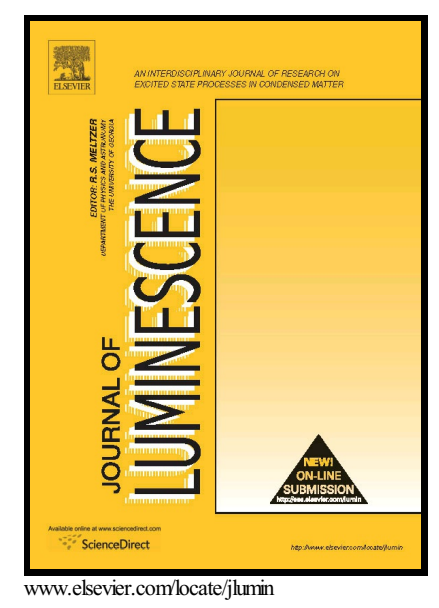

PII: $\quad$ S0022-2313(17)31103-1

DOI: $\quad$ https://doi.org/10.1016/j.jlumin.2017.11.051

Reference: LUMIN15200

To appear in: Journal of Luminescence

Received date: 28 June 2017

Revised date: 12 November 2017

Accepted date: 24 November 2017

Cite this article as: Amit Kumar Prasad and Mayank Jain, Dynamics of the deep red $\mathrm{Fe}^{3+}$ photoluminescence emission in feldspar, Journal of Luminescence, https://doi.org/10.1016/j.jlumin.2017.11.051

This is a PDF file of an unedited manuscript that has been accepted for publication. As a service to our customers we are providing this early version of the manuscript. The manuscript will undergo copyediting, typesetting, and review of the resulting galley proof before it is published in its final citable form. Please note that during the production process errors may be discovered which could affect the content, and all legal disclaimers that apply to the journal pertain. 


\title{
Dynamics of the deep red $\mathrm{Fe}^{3+}$ photoluminescence emission in feldspar
}

\author{
Amit Kumar Prasad ${ }^{1} *$ \& Mayank Jain \\ Center for Nuclear Technologies, Technical University of Denmark DTU Ris $\varnothing$ \\ Campus, Roskilde-4000, Denmark \\ * Corresponding author: amitphotonics@gmail.com
}

\begin{abstract}
We present here new characteristics of the $\mathrm{Fe}^{3+}$ emission in feldspar using a combination of site/energy-selective spectroscopy and time-resolved photoluminescence at cryogenic temperatures. Although the variation in the peak energy of $\mathrm{Fe}^{3+}$ emission has been known to vary across feldspar samples depending on the composition and structure, it has never shown before that this emission can vary dynamically even within a single sample. We show that a strong excitation-energy dependent emission (EDE) occurs in $\mathrm{Fe}^{3+}$ for non-resonant excitation at cryogenic temperatures; this characteristic can be exploited for site discrimination within a complex feldspar sample. The radiative relaxation in $\mathrm{Fe}^{3+}\left({ }^{4} \mathrm{~T}_{1} \rightarrow{ }^{6} \mathrm{~A}_{1}\right)$ is shown to be a dynamic process depending on whether the ion is excited using resonant or non-resonant transition. We suggest that during resonant excitation, the excited state of $\mathrm{Fe}^{3+}$ undergoes a slow energytransfer, possibly a spin-lattice interaction, a process that leads to a non-first order effect in the radiative relaxation process.
\end{abstract}

\section{Introduction}

Feldspar is the most widely available mineral in the Earth's crust (>50\% of crustal rocks). It is a tectosilicate comprising of a framework of $\mathrm{AlO}_{4}$ and $\mathrm{SiO}_{4}$ tetrahedra, and depending on the cation composition may occur in alkali feldspar series $\left(\mathrm{KAISi}_{3} \mathrm{O}_{8}-\mathrm{NaAlSi}_{3} \mathrm{O}_{8}\right)$ or plagioclase feldspar series $\left(\mathrm{NaAlSi}_{3} \mathrm{O}_{8}-\mathrm{CaAl}_{2} \mathrm{Si}_{2} \mathrm{O}_{8}\right)$. Optically, feldspar is a direct, wide band gap material $(\sim 7.7 \mathrm{eV})[1]$. Various impurity atoms and structural defects create local energy states, within the band gap, some of which participate in luminescence emissions from the ultraviolet (UV) to

\footnotetext{
${ }^{1}$ Present address: Schulich Faculty of Chemistry and Solid State Institute Technion - Israel Institute of Technology, Haifa, 32000, Israel
} 
the near infrared (IR) upon different modes of excitation [2,3]. These optical properties of feldspar allow its use in optical dating relevant to last $0.5 \mathrm{Ma}$; the technique relies on measurement of electrons trapped within the defects during the geological time using, for e.g., using optically stimulated luminescence [4-6]. The critical developmental aspects of the OSL method, such as improvement in accuracy and precision, increasing the age range, and ease and reliability of signal measurement, all require detailed understanding of the underlying mechanisms that generate luminescence [6-8].

There are many techniques to induce luminescence in feldspar, such as, thermoluminescence (TL), optically stimulated luminescence (OSL) or infrared stimulated luminescence (IRSL), cathodoluminescence $(\mathrm{CL})$, radioluminescence $(\mathrm{RL})$ and photoluminescence (PL) (see review by Krbetschek et al. [2]). Techniques such as TL, CL, RL and OSL require exposure to ionizing radiations, typically high energy ( $\mathrm{keV}-\mathrm{MeV}$ range) electrons or photons, and are based on electron-hole recombination across different sites (or defects) in the lattice. In contrast, PL is induced from photons with energies smaller than the bandgap, which allow site/energy selective probing of a particular defect and its excited state interaction with the host lattice $[3,6]$. Together these techniques provide a powerful tool to understand luminescence behaviour of unknown defects in natural minerals.

The focus of this study is to obtain a detailed understanding of the deep red emission $(\sim 1.7 \mathrm{eV})$, which is well known to arise from the $\mathrm{Fe}^{3+}$ ion in feldspar [9-11]. This ion substitutes for $\mathrm{Al}^{3+}$ in the tetrahedral sites in the feldspar lattice, and it participates in processes involving ionising radiation, e.g., CL and TL $[12,13]$. Spectroscopic investigations have revealed the energy levels of $\mathrm{Fe}^{3+}$ ion in feldspar [14-17]. The emission peak of $\mathrm{Fe}^{3+}$ is generally located between 680-740 $\mathrm{nm}(1.82-1.67 \mathrm{eV})$ depending on the composition $([2,16]$. This emission occurs from a spin forbidden ${ }^{4} \mathrm{~T}_{1} \rightarrow{ }^{6} \mathrm{~A}_{1}$ transition within the $\mathrm{d}^{5}$ electrons of the ion. Brooks et al. [9] reported variations in the $\mathrm{CL} / \mathrm{RL}$ emission of $\mathrm{Fe}^{3+}$ on temperature as a result of changing $\mathrm{Fe}-\mathrm{O}$ bond length due to potassium ions. It has been suggested that $\mathrm{Fe}^{3+}$ emission peak varies both in alkali feldspar and plagioclase feldspar depending on the $\mathrm{K} / \mathrm{Na}$ ratio or the $\mathrm{Ca} / \mathrm{Na}$ ratio, respectively [12]. Furthermore, the site occupancy of $\mathrm{Fe}^{3+}$ in alkali feldspars plays an important role in determining the emission wavelength. For example, $\mathrm{Fe}^{3+}$ substitutes for $\mathrm{T}_{1}$ tetrahedral site in ordered feldspar leading to red emission, or both $\mathrm{T}_{1}$ and $\mathrm{T}_{2}$ sites in disordered feldspar lattice 
leading to IR emission [12, 18, 19]. The main causes of these variations are the crystal field effect and the changing bond lengths of Fe-O, both of which modify the ${ }^{4} \mathrm{~T}_{1} \rightarrow{ }^{6} \mathrm{~A}_{1}$ transition energy level.

In terms of dosimetric research, it has been well documented that red TL undergoes very strong thermal quenching [13], and it has been suggested that this signal does not suffer from anomalous fading, both in TL [20] and IRSL [21-23]. There are two different views on the role of $\mathrm{Fe}^{3+}$ in luminescence dosimetry. One suggestion is that $\mathrm{Fe}^{3+}$ captures a free electron during ionising irradiation (converting to $\mathrm{Fe}^{2+}$ ), and a subsequent capture of a hole during heating results in an $\mathrm{Fe}^{3+}$ excited state, which then relaxes to give the red emission [e.g., 13]. Recently, Jain et al. [7] based on their investigations on IRSL kinetics proposed an alternative view: $\mathrm{Fe}^{3+}$ captures a free hole during ionising irradiation (leading to $\mathrm{Fe}^{4+}$ ), and a subsequent capture of free electron during the IRSL process gives rise to the excited state of $\mathrm{Fe}^{3+}$, which subsequently relaxes to emit deep red photons.

Despite a great interest in $\mathrm{Fe}^{3+}$, both in dosimetric and mineralogical studies [2, 9, 16, 20-23], the details of the $\mathrm{Fe}^{3+}$ system in feldspar are still missing. In contrast to the emission characteristics [2], the excitation energy dependence of the $\mathrm{Fe}^{3+}$ emission has not been studied in great detail. There is a general lack of understanding of: a) the site dependence of $\mathrm{Fe}^{3+}$ emission in a single sample (previous studies have focussed on relative spectral variations across samples of different compositions), b) the dependence of emission peak on temperature and excitation energy, c) the dependence of relaxation lifetime on temperature and/or excitation energy (resonant vs. nonresonant). Understanding these aspects will provide insights into the interactions of $\mathrm{Fe}^{3+}$ with the host lattice, and potentially lead to a better exploitation of this important emission in luminescence dosimetry.

Here we use site/energy-selective spectroscopy combined with low temperature measurements, and time-resolved photoluminescence studies to understand the dynamics of the $\mathrm{Fe}^{3+}$ emission in feldspar. We show that this emission not just varies across samples, as shown in the previous studies, $[12,16,18,19]$, but it can also vary significantly within the same sample. We also give insight into the spin forbidden relaxation mechanism in $\mathrm{Fe}^{3+}$.

\section{Experimental}


We measured $\mathrm{Fe}^{3+}$ emission and excitation spectrum in 11 different feldspar samples listed in Table 1. The sample were granular ( 90-180 $\mu \mathrm{m}$ diameter grains) except for R28 which is a single crystal of $2 \times 2 \times 2 \mathrm{~mm}$ size. The Fe content of these samples was measured using Thermo X-series II - Quadrupole inductively coupled plasma mass spectrometry (ICP-MS), and their bulk composition $(\mathrm{KF}, \mathrm{NaF}$ and $\mathrm{CaF}$ ) determined using the Ris $\emptyset$ X-ray fluorescence (XRF) system. The $\mathrm{K} / \mathrm{Na} / \mathrm{Ca}$ content of each sample (Table 1) represents the average composition of the entire sample area on an aliquot used for PL measurement. The sample geometry during the PL was designed such that there is full, uniform illumination of the aliquot.

All the excitation, emission, and luminescence decay measurements were carried out using Ris $\varnothing$ Station for CryOgenic LUminecence Research (COLUR) at DTU Nutech, Technical University of Denmark. COLUR consists of Horiba Fluorolog-3 spectrofluorometer, a closed-loop helium cryostat, and several detectors and excitation sources for multi-probe measurements. The detectors used are photomultiplier tube (PMT) and cooled charged coupled devices (CCD), coupled to monochromator gratings or band pass filters. Various excitation sources include Xray tube (40 kV, $100 \mu \mathrm{A}$ copper anode, $3 \mathrm{~ms}$ shutter speed), and continuous wave or pulsed Xe lamp, lasers and light emitting diodes. Sample is mounted on the cold finger of the cryostat, and the temperature can be controlled between $7-300 \mathrm{~K}$ under vacuum $\left(2.5 \times 10^{-5} \mathrm{mb}\right)$ using the Lakeshore temperature control unit (model 335).

Specifically, PL emission spectra were measured using a liquid nitrogen cooled CCD detector. The excitation spectra were measured with a Cherny-Turner type monochromator in front of a photo multiplier tube (PMT) detector. The excitation wavelength was selected using the double grating Cherny-Turner type monochromator from a 450W Xenon lamp and a $610 \mathrm{~nm}$ long-pass filters was placed at the detection side to avoid any stray light. The results presented here are corrected for the instrument response and excitation flux. Phosphorescence decay was measured using a $450 \mathrm{~W}$ Xenon lamp operated in a pulsed mode, and detection of luminescence using a PMT with a multi-channel scaler. The integration time for phosphorescence collection was 100 $\mathrm{ms}$ and the initial delay was $0.05 \mathrm{~ms}$. The X-ray tube was used for used for investigating sample's response to ionizing dose. X-ray irradiations were done at $295 \mathrm{~K}$. 


\section{Results and Discussion}

Our investigations into the temperature and excitation energy dependence of the $\mathrm{Fe}^{3+}$ emission, and its decay characteristics are described below.

\section{1. $\mathrm{Fe}^{3+}$ excitation and emission: general characteristics}

The emission spectra of 11 different feldspars samples (Table 1), measured at room temperature, are shown in Figure 1 (a). The samples were excited with $4.67 \mathrm{eV}$ photons, and the deep red emission was recorded between 1.4 and $2.1 \mathrm{eV}$. The corresponding excitation spectra were measured by scanning the excitation energy from 1.9 to $4.9 \mathrm{eV}$ while detecting the emission at the peak emission wavelength of the individual samples (Figure 1 (b)). We observe that the emission peak position shifts across samples, from $1.67 \mathrm{eV}$ in $\mathrm{R} 58$ to $1.8 \mathrm{eV}$ in R64; the majority of the samples have their emission peak at $\sim 1.7 \mathrm{eV}$. This emission arises from the forbidden ${ }^{4} \mathrm{~T}_{1}$ $\rightarrow{ }^{6} \mathrm{~A}_{1}$ transition $[11,14]$. The excitation spectra in Figure 1(b) show several resonant excitation peaks in the region 2.5 to $3.4 \mathrm{eV}$, and a non-resonant continuum up to $>4.5 \mathrm{eV}$. Similar to the emission peak positon, some (weak) resonant excitation peaks, as well as the rise of the nonresonant continuum vary slightly with feldspar composition.

Earlier studies have shown that the peak position of $\mathrm{Fe}^{3+} \mathrm{PL}$ emission is related to the mineralogical composition [16-18]. However, we do not observe any obvious correlation between the emission peak position and the $\mathrm{K}$ content in our samples (Table 1); this suggests that the peak energy variations in our samples may perhaps be controlled by other factors such as the structural state of feldspar and the tetrahedral site dependence of $\mathrm{Fe}^{3+}$.

We chose sample R56 (alkali feldspar with similar KF and NaF content) as a representative sample for detailed investigations; its emission peak position is similar to that for samples R44, R50, R57 and R63, and lies in close vicinity of the emission peaks of R42, R43, R47 and R49 (Figure 1(a)). The purpose of these detailed measurements was to understand the temperature and excitation-energy dependence of the $\mathrm{Fe}^{3+}$ emission.

\subsection{Temperature and excitation-energy dependence of the $\mathrm{Fe}^{3+}$ emission in $\mathrm{R} 56$}

Figure 2 gives a closer view of the excitation and the emission spectrum of R56 from Figure 1; the PL intensity is plotted on the log (intensity) scale for the excitation spectrum (right) and on 
the linear scale for the emission spectrum (left). The excitation spectrum was obtained by fixing the emission at $1.7 \mathrm{eV}$, while the emission spectrum was obtained using $4.67 \mathrm{eV}$ excitation. The inset of Figure 2 shows the energy level diagram for the isolated $\mathrm{Fe}^{3+}$ defect in the host $[14,15]$. It can be seen that there are several excitation levels but the radiative relaxation occurs only from the lowest ${ }^{4} \mathrm{~T}_{1}(\mathrm{G})$ level; the exact energies depend upon the crystal field in the sample. Four of these transitions are marked against the dominant emission and excitation peaks in R56 (Figure $2)$. The non-resonant continuum in the excitation spectrum shows an exponential dependence on energy from $\sim 3.8$ to $4.6 \mathrm{eV}$.

$\mathrm{Fe}^{3+}$ emission is known to show a strong thermal quenching at room temperature [13-15]; however, there are not many studies of changes in emission energy as a function of temperature. A shift in the emission energy with temperature could occur because of changing crystal field due to shrinkage/expansion, and/or participation of different vibrational levels. In order to examine the effect of cooling on R56, we measured PL emission spectra at different temperatures while exciting with $4.67 \mathrm{eV}$ photons (Figure 3(a)). We observe a gradual blue shift, peak widening and a decrease in the emission intensity with temperature. The emission shift has a magnitude of $0.25 \mathrm{eV}$ from room temperature to $7 \mathrm{~K}$. The most plausible reasons for the peak shift and widening are phonon interactions and an increasing role of the higher energy vibrational levels at higher temperatures. We do not see any blue shift in the $\mathrm{Fe}^{3+}$ emission on cooling, a typical temperature dependent effect is semiconductors [Varshni effect; 24].

The effect of changing the excitation energy on the emission peak energy of $\mathrm{Fe}^{3+}$ has never been, to our knowledge, investigated before. Figure 3(b) shows the emission spectra measured at 295 $\mathrm{K}$, as the excitation energy was varied from 4.0 to $4.86 \mathrm{eV}$. Similar to the orange-green emission in feldspar [3, 25], we observe an excitation-energy dependent emission (EDE) ${ }^{2}$ (Figure 3(b)); there is a red shift in the emission peak with a decrease in the excitation energy (apparently similar to the red edge effect) $[3,25,26]$. Figure 4(a) shows the summary of the peak shift as a function of excitation energy in both $\mathrm{K}$ rich alkali feldspar (R56; black 'plus' symbol) and a Na rich alkali Feldspar (R58; red 'cross' symbol) samples at room temperature. The reference excitation spectra for these samples are also plotted using the same colour scheme (black for R56 and red for R58); these excitation spectra were obtained while fixing the emission at $1.7 \mathrm{eV}$.

\footnotetext{
${ }^{2}$ EDE: Excitation-Energy Dependent Emission. This effect apparently violates the Kasha's rule that the emission occurs from the lowest electronic/vibrational excited state and is independent of the excitation energy [see 26].
} 
Figure 4 (b) is similar to Figure 4(a) except that the full width at half maximum (FWHM) of the PL emission peaks are plotted here. Figure 4(a) suggests that the EDE occurs both in Na and K rich feldspar (although with different magnitudes) in the non-resonant excitation continuum; there is a red shift of $\sim 0.02 \mathrm{eV}$ in $\mathrm{R} 56$ and $\sim 0.04 \mathrm{eV}$ in R58. There is also suggestion for a minor EDE across the resonance peak at $3.35 \mathrm{eV}$ (Figure 4(a)). The FWHM decreases with an increase in the non-resonant excitation energy (Figure 4(b)).

To gain insight into this EDE, we repeated the same experiment as described for Figure 4, but at ten different measurement temperatures between 7 and $295 \mathrm{~K}$. These temperature-dependent measurements were carried out only for the K-feldspar sample (R56). Figure 5 shows the summary of the $\mathrm{Fe}^{3+}$ emission peak as a function of excitation energy and temperature; peak position and FWHM are shown in Figure 5(a), and Figure 5(b), respectively, for selected temperature (to avoid cluttering). Normalised peak area, with respect to the peak area at $7 \mathrm{~K}$, is plotted in Figure 5(c).

The EDE is significantly enhanced with cooling in the non-resonant excitation region (4.0 -4.86 $\mathrm{eV}$; Figure 5(a)). Furthermore, the temperature dependent red shift strongly varies with excitation energy in the region 3.9-4.4 eV; but it is nearly constant for resonant excitation (2.7$3.4 \mathrm{eV})$ and the high-energy end of non-resonant excitation $(4.5-5.0 \mathrm{eV})$ (Figure 5(a).

There occurs a significant overall reduction in the FWHM with sample cooling both in the resonant and non-resonant regions; this is the same as the temperature dependent phonon effect discussed for Figure 3(a). For the non- resonant region, the emission shows a systematic narrowing with excitation energies at room temperature, but this trend becomes non monotonic (peak shaped) at low temperatures (Figure 5(b)).

Figure 5(c) shows that thermal quenching exists (reduction in the PL emission area with a rise in temperature) for all the excitation energies but the extent of thermal quenching varies with the excitation energy.

To our knowledge, such effects of $\mathrm{Fe}^{3+}$ emission on excitation energy and temperature have not been reported earlier. In summary, this section makes several new observations: a) a strong phonon effect, leading to a red shift and narrowing of $\mathrm{Fe}^{3+}$ PL emission, dependent on the mode of excitation (resonant vs. non-resonant), b) an excitation-energy dependent emission, which is 
enhanced with sample cooling, and c) different thermal quenching behaviour of the $\mathrm{Fe}^{3+}$ emission depending on the excitation energy.

As discussed in the introduction, the emission peak in $\mathrm{Fe}^{3+}$ has been known to shift across samples because of variation in composition or the site occupancy of $\mathrm{Fe}^{3+}$. The sample $\mathrm{R} 56$ contains roughly equal amounts of $\mathrm{KF}$ and $\mathrm{NaF}$ content; it is conceivable that EDE observed in our data arises from changes in the relative excitation of the $\mathrm{T}_{1}$ and $\mathrm{T}_{2}$ sites, or separate $\mathrm{K}$ and $\mathrm{Na}$ feldspar sub-phases within the sample. To confirm this hypothesis, we chose a pure $\mathrm{K}$ feldspar sample (R28) (Table 1). The emission spectra measured with three different excitation energies are shown in Figure 6. Unlike sample R56, we do not see any EDE in R28. The three peaks are very similar and can be described by a single Gaussian function. Figure 6 inset shows a single peak fitting for the case of $4.86 \mathrm{eV}$ excitation. These data from R28 suggest that sample R56 must contain mixed phases or two different sites of $\mathrm{Fe}^{3+}$ with different crystal field around them; what we observe is a summed PL emission from both the sites. On non-resonant excitation, the relative strength of the two emissions changes, giving rise to an apparent peak shift in the summed signal. With this insight, we re-analysed the data reported in Figures 3, 4 and 5 with a two-peak model, representing two $\mathrm{Fe}^{3+}$ sites or compositional sub-phases within the same sample. For convenience, we will refer to this as the two-site model.

\subsection{Testing the two- $\mathrm{Fe}^{3+}$-site model in $\mathrm{R56}$}

Figure 7(a) shows the temperature dependent FWHM of the $\mathrm{Fe}^{3+}$ emission for all the excitation energies (4.0 - $4.86 \mathrm{eV})$ and measurement temperatures; note that Figure 5(b) represents only part of this excitation-emission-temperature matrix to avoid a clutter. The FWHM data show an apparent peak trend which begins to emerge at temperatures below $200 \mathrm{~K}$. At temperatures below $100 \mathrm{~K}$ there exists a clear peak in the FWHM at $\sim 4.3 \mathrm{eV}$ excitation energy. These FWHM data support the two-site model, which becomes especially clearer at low temperatures because of reduction in the phonon induced broadening of the individual peaks. Thus, the end members on the excitation energy scale represent dominant excitation of a single $\mathrm{Fe}^{3+}$ site resulting in a narrow FWHM, whereas around $4.3 \mathrm{eV}$ both the sites are excited with similar efficiency resulting in a wide FWHM. The overall result is a peak shaped variation in FWHM due to overlapping of the two peaks as a function of excitation energy. At higher temperatures, these 
differences are systematically dampened because of a significant phonon induced broadening as well as thermal quenching of the individual peaks.

With this qualitative confirmation of the applicability of two-site model to R56, we performed peak fitting of the emission spectra obtained from $4.86 \mathrm{eV}$ excitation at $295 \mathrm{~K}$ (see Figure 7(b)). As expected, unlike R28, the data from R56 were best described by a sum of two Gaussian peaks. As a further qualitative test of the two peak model, we measured the excitation spectrum of $\mathrm{R} 56$ at $7 \mathrm{~K}$ while fixing the emission window at $1.79 \mathrm{eV}$ or $1.57 \mathrm{eV}$, thereby, trying to obtain site specific excitation from a complex sample. The data are shown in Figure 7(c). The $7 \mathrm{~K}$ temperature was used to maximise the separation of the two peaks (Figure 7(a)) and thus obtain a significant EDE (Figure 5(a)). We observe a slight variation in the resonant peaks at $\sim 2.9 \mathrm{eV}$ and a clear shift of the non-resonant excitation, supporting the view that different sites of $\mathrm{Fe}^{3+}$ are present in sample R56.

Finally, to make a quantitative test of the two-peak model, we tried to fit a sum of two Gaussian peaks to all our excitation-emission-temperature data from R56. The positions of the peak components P1 and P2 are shown in Figure 7(d) for the data measured at $7 \mathrm{~K}$ and $295 \mathrm{~K}$ only (to avoid cluttering); as can be seen in the $7 \mathrm{~K}$ data, although, peak fitting helps to reduce the EDE, it does not completely eliminate it. We also tried to force-fit two shared peaks to all the data, but this attempt was unsuccessful; the obtained peak fits were poor suggesting that two common peaks do not completely describe the EDE in R56. Thus, it is possible that other effects may also play a role leading to an inhomogeneous broadening of the $\mathrm{Fe}^{3+}$ emission in $\mathrm{R} 56$; this needs further investigations. Nonetheless, it is clear from our analysis that the two-site model is a good first order approximation to explain the EDE in the $\mathrm{Fe}^{3+}$ emission in feldspar.

\subsection{Time resolved PL data}

We examined the temperature dependent lifetime of $\mathrm{Fe}^{3+}$ emission at $1.7 \mathrm{eV}$ with excitations at $4.77 \mathrm{eV}$ (non-resonant) and $3.29 \mathrm{eV}$ (resonant); the data are shown in Figure 8(a), and 8(b), respectively, and fitting parameters are summarised in Table 2. All the phosphorescence data show a slow decay confirming the spin-forbidden radiative transition in $\mathrm{Fe}^{3+}$ [e.g., 3]. Furthermore, there is a systematic decrease in the average lifetime with temperature, confirming the thermal quenching mechanism in $\mathrm{Fe}^{3+}$. The lifetime decreases because of increase in nonradiative relaxation from the higher energy vibrational levels at higher temperatures. 
The resonant PL decay data could not be fitted to either a single exponential, or a sum of two exponential functions as the data after $\sim 4 \mathrm{~ms}$ shows an unusual kink (see Figure 8(b); this perhaps suggests a relatively short lived build-up process before the radiative relaxation begins to dominate. We interpret that relaxation from the resonant (excited) state may be a two step process: a) energy transfer between the excited state and the lattice, which results in reorientation of the spin (lattice-spin interaction), and b) excited to ground state relaxation. The non-resonant data on the other hand show clear single exponential decay until the temperature of $200 \mathrm{~K}$, while at room temperature the decay shows two exponentials. As inferred from Figure 7(a), the 4.77 $\mathrm{eV}$ photons are likely to excite only one of the $\mathrm{Fe}^{3+}$ sites at low temperature, but both the $\mathrm{Fe}^{3+}$ sites at room temperature. Thus, the single exponential and double exponential PL decay in Figure $8(\mathrm{a})$, is likely related to the site-dependent excitation of $\mathrm{Fe}^{3+}$.

The lifetime values $\left(\tau_{4.77}\right)$ derived from exponential fitting of the non-resonant data (for $4.77 \mathrm{eV}$ excitation) are summarised in Table 2. For the resonant data (3.29 eV excitation), we divided the curve in two parts (Figure $8(\mathrm{c})$ ), before and after the kink, and fitted them individually to exponential functions; the lifetimes are in summarised in Table 2 as $\tau_{3.3}{ }^{*}$ and $\tau_{3.3}$, respectively. It can be seen that the $\tau$ values for the resonant and non-resonant excitations $\left(\tau_{3.3}\right.$ and $\left.\tau_{4.77}\right)$ are almost same; these must, therefore, reflect the lifetime of the radiative transition $\left({ }^{4} \mathrm{~T}_{1} \rightarrow{ }^{6} \mathrm{~A}_{1}\right)$. The $\tau_{3.3} *$ values (before the kink) on the other hand, in our view, possibly reflect the time scale involved in interaction between the excited state and the crystal through energy transfer. A more sophisticated interaction model than a simple single exponential fitting is needed to determine the exact lifetime of this process.

\subsection{X-ray irradiation (dose) dependence of the $\mathrm{Fe}^{3+}$ emission}

Finally, we tested if the $\mathrm{Fe}^{3+}$ PL emission can be exploited to measure ionising radiation dose in feldspar. We expect that the $\mathrm{Fe}^{3+}$ concentration should decrease with radiation dose because of the conversion of this species to either $\mathrm{Fe}^{2+}[13]$ or $\mathrm{Fe}^{4+}$ [7]. We subjected an aliquot of R56 to different durations of X-ray exposure, while we intermittently measured the $\mathrm{Fe}^{3+}$ emission spectrum using $3.29 \mathrm{eV}(377 \mathrm{~nm})$ optical stimulation. Figure 9 shows the results; we do not observe any clear dosimetric PL signal up to 70 minutes of X-ray exposure (approximately 250 Gy, dose rate of X-ray: $0.06 \mathrm{~Gy} / \mathrm{s}$ ). This is likely because of a huge background arising from a 
large concentration of $\mathrm{Fe}^{3+}$ in feldspars (see Table 1), where irradiation changes the ionic state of only a negligible fraction of $\mathrm{Fe}^{3+}$.

\subsection{Discussion - dynamic behaviour of $\mathrm{Fe}^{3+}$ in feldspar}

We carried out site selective-spectroscopy of $\mathrm{Fe}^{3+}$ in a variety of samples, and chose an alkali feldspar sample for detailed investigations (R56). We observe that the $\mathrm{Fe}^{3+}$ emission peak varies significantly from sample to sample.

We see a systematic red shift and a reduction in FWHM in the $\mathrm{Fe}^{3+}$ emission with cooling. The red shift in peak position on cooling is dependent on the excitation energy in the $3.9-4.4 \mathrm{eV}$ range; this shift may be as much as $0.09 \mathrm{eV}$, for example, for non-resonant excitation at $3.9 \mathrm{eV}$ (Figure 5(a)). But, we also see a second component of red shift that is independent on the excitation energy; this is nearly constant, of the order of about $\sim 0.04 \mathrm{eV}$, and is visible for resonant excitation (2.7- 3.4 eV) and the high energy (flat) end of non-resonant excitation (4.5$5.0 \mathrm{eV}$ ) (Figure 5(a)). We interpret that the first energy-dependent red-shift component is a result of the two-site model. Different rates of thermal quenching of the two $\mathrm{Fe}^{3+}$ sites give rise to an apparant peak shift. The second component of peak shift which is constant and independent of excitation energy, arises due to relaxation from the lower vibrational levels of the ion on cooling. The latter effect also explains the peak narrowing at lower temperatures.

Our site-selective data for the first time demonstrate heterogeneity of $\mathrm{Fe}^{3+}$ within a single sample. We show a strong excitation dependent emission (EDE), apparently violating Kasha's rule $[25,26]$, in both $\mathrm{K}$ rich and $\mathrm{Na}$ rich alkali feldspar. The EDE effect is systematically enhanced at cryogenic temperatures, which, together with the systematic variation in FWHM as a function of excitation energy and temperature, can be qualitatively explained in terms of different site occupancies of the $\mathrm{Fe}^{3+}$ ion or heterogeneous compositional sub-phases within a feldspar sample. The quantitative evaluation of EDE using two $\mathrm{Fe}^{3+}$ site model (two peak model), although quite successful compared to a single site model, does not fully account for the EDE. A minor excitation-energy dependence is still observed in peak 2 at $7 \mathrm{~K}$ (Figure 7(d)). This second order effect, perhaps suggests that there are more than just two components within the $\mathrm{Fe}^{3+}$ emission, e.g. related to lattice disorder, or that there are other energy interactions in the system related to non-resonant excitation, that are presently not known. 
The exponential dependence of the non-resonant excitation spectrum (Figure 1(b)) may suggest presence of a charge transfer band with an exponential density of states [3, 25]. Such a band may arise due to local defect clustering of $\mathrm{Fe}^{3+}$ ions, thus forming a continuum of excited state levels, or show the effect of the sub-conduction (Urbach) band tail states [27]. However, the insights obtained from the two-site model suggest that the charge transfer band responsible for the nonresonant continuum arises from an amalgamation of the higher energy levels of the both the sites, thus making the non-resonant excitation site selective. The exponential trend of the excitation spectrum may then possibly reflect the behaviour of ionisation cross-section with energy [28].

The phosphorescence decay at room temperature and at $7 \mathrm{~K}$ using resonant or non-resonant excitation suggests that the ${ }^{4} \mathrm{~T}_{1} \rightarrow{ }^{6} \mathrm{~A}_{1}$ transition involves a dynamic interaction between the lattice and $\mathrm{Fe}^{3+}$ ion. During resonant excitation, an additional time step is involved, possibly due to an energy exchange between the excited state and the crystal resulting in a spin reorientation; apparently, the time scale for this process is of the order of milliseconds and it depends on temperature; this is a new insight presented by this study, which deserves further exploration. In the case of non-resonant excitation, this additional step is not involved in the relaxation process; a single exponential decay is observed at low temperatures (from single $\mathrm{Fe}^{3+}$ site), while a double exponential decay is observed at room temperature (from two $\mathrm{Fe}^{3+}$ sites). The non-resonant PL decay data apparently support the existence of a two component $\mathrm{Fe}^{3+}$ system. It appears that lattice-spin interaction may be less important in the non-resonant excitation because of rapid thermalisation within the centre or within the charge transfer band [3]; this is interesting and requires further investigations.

Since EDE is particularly sensitive to sample heterogeneity, a correlation of EDE with the dose measurement in single grains of feldspar can potentially provide an important tool for quality control in optical dating [4-6].

\section{Conclusions}

We report for the first time an excitation-energy dependent emission (EDE) in $\mathrm{Fe}^{3+}$ in feldspar, which is especially prominent for non-resonant excitation at cryogenic temperatures. The red shift in the peak of the emission can be as much as $\sim 0.1 \mathrm{eV}(730-780 \mathrm{~nm}$ ) upon lowering the excitation energy by about $0.6 \mathrm{eV}(275-320 \mathrm{~nm})$. The EDE effect to a large extent can be explained by $\mathrm{Fe}^{3+}$ occupying two different sites within the feldspar lattice or within different 
compositional sub-phases. This inference is supported by temperature dependence of the phosphorescence decay kinetics for non-resonant excitation. Interestingly, we observe an additional time constant involved in ${ }^{4} \mathrm{~T}_{1} \rightarrow{ }^{6} \mathrm{~A}_{1}$ relaxation during resonant excitation, which we tentatively attribute to transient energy transfer between the ion and the lattice. Our results suggest that EDE (especially at cryogenic temperatures) because of its site-selective nature could be a powerful tool to map mineralogical/structural complexity within a single feldspar sample.

\section{Acknowledgement}

We thank Prof. Adrian Finch and Dr. Nigel Poolton for discussions during the early phase of this work. We thank Dr. Jan-Pieter Buylaert for sediment samples, Dr. Sumiko Tsukamoto for the R63 and R64 samples, Dr. Poolton for R28 sample, Dr. Myungho Kook for XRF measurements, Dr. Per Roos for ICP-MS measurements and Ms. Louise Maria Helsted for sample preparation. We are thankful to two anonymous reviewers for their critical comments on the manuscript.

\section{References:}

[1] A. E. R. Malins, N. R. J. Poolton, F. M. Quinn, O. Johnsen, P. M. Denby, Luminescence excitation characteristics of $\mathrm{Ca}, \mathrm{Na}$ and $\mathrm{K}$-aluminosilicates (feldspars) in the stimulation range 5-40 eV: determination of the band-gap energies, J. Phys. D: Appl. Phys., 37 (2004) 1439-1450

[2] M. R. Krbetschek, J. Götze, A. Dietrich, T. Trautmann, Spectral information from minerals relevant for luminescence dating, Radiation Measurements, 27(5) (1997), 695-748.

[3] A. K. Prasad, T. Lapp, M. Kook, M. Jain, Probing luminescence centers in Na rich feldspar, Radiation Measurements, 90(2016), 292-297.

[4] D. J. Huntley, D. I. Godfrey-Smith, M. L. W. Thewalt, Optical dating of sediments, Nature 313, 105-107 (1985).

[5] G. Hütt, I. Jaek, J. Tchonka, Optical dating: K-feldspars optical response stimulation spectra, Quaternary Science Reviews, 7 (1988), 381-385.

[6] A. K. Prasad, N. R. J. Poolton, M. Kook, M. Jain, Optical dating in a new light: A direct, non-destructive probe of trapped electrons, Scientific Reports, 7(2017), Article number: 12097. 
[7] M. Jain , R. Sohbati, B. Guralnik, A. S. Murray, M. Kook, T. Lapp, A. K. Prasad, K. J. Thomsen, J. P. Buylaert, Kinetics of infrared stimulated luminescence from feldspars, Radiation Measurements, 81(2015), 242-250.

[8] M. Jain, C. Ankjærgaard, Towards a non-fading signal in feldspar: insight into charge transport and tunnelling from time-resolved optically stimulated luminescence, Radiation Measurements, 46(3) (2011), 292-309.

[9] R. J. Brooks, A. A. Finch, D. E. Hole, P. D. Townsend, Z. L. Wu, The red to near-infrared luminescence in alkali feldspar, Contributions to Mineralogy and Petrology, 143(4) (2002) 484494.

[10] J. E. Geake, G. Walker, D. J. Telfer, A. A. Mills, The cause and significance of luminescence in lunar plagioclase, Philosophical Transactions of the Royal Society of London A: Mathematical, Physical and Engineering Sciences, 285 (1327) (1977), 403-408.

[11] D. J. Telfer, G. Walker, Optical detection of $\mathrm{Fe}^{3+}$ in lunar plagioclase, Nature, 258(5537) (1975), 694-695.

[12] M. R. Krbetschek, J. Götze, G. Irmer, U. Rieser, T. Trautmann, The red luminescence emission of feldspar and its wavelength dependence on $\mathrm{K}, \mathrm{Na}, \mathrm{Ca}-$ composition, Mineralogy and Petrology, 76(3-4) (2002), 167-177.

[13] R. Visocekas, C. Barthou, P. Blanc,Thermal quenching of far-red $\mathrm{Fe}^{3+}$ thermoluminescence of volcanic K-feldspars, Radiation Measurements, 61(2014), 52-73.

[14] W. B. White, M. Matsumara, D. G. Linnehan, T. Furukawa, B. K. Chandrasekhar, Absorption and luminescence of $\mathrm{Fe}^{3+}$ in single-crystal orthoclase, Am. Mineral. 71 (14) (1986)15-19.

[15] N. R. J. Poolton, L. Bøtter-Jensen, G. A. T. Duller, Thermal quenching of luminescence processes in feldspars, Radiation Measurements, 24(1) (1995), 57-66.

[16] N. R. J. Poolton, L. Bøtter-Jensen, O. Johnsen, On the relationship between luminescence excitation spectra and feldspar mineralogy, Radiation measurements, 26(1) (1996), 93-101. 
[17] N. R. J. Poolton, B. Mauz, A. Lang, M. Jain, A. E. R. Malin, Optical excitation processes in the near band-edge region of $\mathrm{KAlSi}_{3} \mathrm{O}_{8}$ and $\mathrm{NaAlSi}_{3} \mathrm{O}_{8}$ feldspar, Radiation measurements, 41(5) (2006), 542-548.

[18] M. A. Short, Polarization effects in the excitation and emission of $\mathrm{Fe}^{3+}$ in orthoclase and their relevance to the determination of lattice sites of unknown defects, J. Phys.: Condens. Matter, 17 (2005) 205-220.

[19] A. A. Finch, J. Klein, The causes and petrological significance of cathodoluminescence emissions from alkali feldspars, Contributions to Mineralogy and Petrology, 135(2-3) (1999) 234-243.

[20] J. C. Zink, R. Visocekas, Datability of sanidine feldspars using the near-infrared TL emission, Radiation Measurements, 27(2) (1997), 251-261.

[21] M. Fattahi, S. Stokes, Red luminescence from potassium feldspar for dating applications: a study of some properties relevant for dating, Radiation Measurements, 37(6) (2003), 647-660.

[22] M. Fattahi, S. Stokes, M. Lamothe, Red luminescence emission from potassium feldspars stimulated by infrared, Ancient TL, 22(2) (2004) 35-44.

[23] S. Stokes, M. Fattahi, Red emission luminescence from quartz and feldspar for dating applications: an overview, Radiation Measurements, 37(4) (2003), 383-395.

[24] Y.P. Varshni, Temperature dependence of the energy gap in semiconductors, Physica, 34 (1967), 149-154.

[25] A. K. Prasad, M. Jain, Breakdown of Kasha`s Rule in a Ubiquitous Naturally Occurring, Wide Bandgap Aluminosilicate Mineral (Feldspar), (Under review), 2017.

[26] A.P. Demchenko, The red-edge effects: 30 years of exploration, Luminescence, 17(1) (2002) 19-42.

[27] S. John, C. Soukoulis, M. H. Cohen, E. N. Economou, Theory of electron band tails and the Urbach optical-absorption edge, Physical review letters, 57(14) (1986), 1777.

[28] K.W. Boer, Survey of semiconductor physics: Electrons and other particles in bulk semiconductors, Van Nostrand Reinhold, New York. 


\section{Figure captions}

Figure 1 (a) emission and (b) excitation spectra of 11 different feldspars samples measured at room temperature. The emission spectra were obtained using the excitation at $4.67 \mathrm{eV}$, whereas the excitation spectra were obtained by fixing the detection window at the peak emission for the individual samples.

Figure 2. A detailed view of the emission and excitation spectra of sample R56. The inset shows the energy level diagram of an isolated $\mathrm{Fe}^{3+}$ within a host lattice. The main transitions following this diagram are marked against the peak data.

Figure 3 (a) PL emission spectra of R56 at different temperatures using a $4.67 \mathrm{eV}$ excitation. (b) PL emission spectra of R56 measured at $295 \mathrm{~K}$ using different excitation energies.

Figure 4 (a) Dependence of the $\mathrm{Fe}^{3+}$ PL emission peak position (right ordinate) on the excitation energy for R56 (K rich feldspar) and R58 (Na rich feldspar) at $295 \mathrm{~K}$. The reference excitation spectrum (left ordinate) is plotted for each sample using the same colour as the peak position. (b) Same as (a) but showing the full width half maximum (FWHM; right ordinate) of the $\mathrm{Fe}^{3+}$ emission peak.

Figure 5 (a) Dependence of the $\mathrm{Fe}^{3+} \mathrm{PL}$ emission peak position on the excitation energy and measurement temperature for R56. The left ordinate refers to the excitation spectrum (dashed curve), while the right ordinate refers to the emission peak energies (coloured symbols) corresponding to different excitation energies (b) Same as (a) but for FWHM, plotted as coloured symbols (c) Dependence of integrated PL peak intensity on measurement temperature for different excitation energies. 
Figure 6. PL emission of R28 for three different excitation energies. The inset shows the fit of the $4.86 \mathrm{eV}$ data to a single Gaussian peak.

Figure 7 (a) Temperature dependent FWHM of the $\mathrm{Fe}^{3+}$ PL emission for different excitation energies. (b) PL emission spectrum at $295 \mathrm{~K}$ ( $4.86 \mathrm{eV}$ excitation) fitted to a sum of two Gaussian peak functions. (c) Excitation spectrum of R56 at $7 \mathrm{~K}$ for detection fixed at $1.79 \mathrm{eV}$ or $1.57 \mathrm{eV}$. (d) Dependence of the two peak components (P1 and P2) of the $\mathrm{Fe}^{3+}$ emission on the excitation energy and the measurement temperature ( $295 \mathrm{~K}$ or $7 \mathrm{~K})$.

Figure 8. Temperature dependent PL decay measurements using (a) non-resonant excitation at $4.77 \mathrm{eV}$, and (b) resonant excitation at $3.29 \mathrm{eV}$. The emission detection was fixed at $1.7 \mathrm{eV}$. (c) Separation of the phosphorescence decay curves before (left) and after (right) the kink shown in (b). The curves are normalised to begin at unity and are fitted with single exponential function to derive temperature dependent lifetime.

Figure 9. X-ray irradiation dependence of $\mathrm{Fe}^{3+} \mathrm{PL}$ emission using a $3.29 \mathrm{eV}$ excitation energy. The inset shows the area under the PL curve vs. X-ray irradiation time.

Table 1: Feldspar samples used in this studies, their bulk composition as determined by XRF and Fe content evaluated using ICP-MS. S represents feldspars of sedimentary origin, and M represents museum samples. Here, KF: K-Feldspar, NaF: Na-Feldspar, CaF: Ca-Feldspar, \#: single crystal sample, u/k: unknown and \$: Not measured. The bold letters indicates that the $\mathrm{Fe}^{3+}$ emission characteristics of this samples was explored in detail (see text).

\begin{tabular}{|c|c|c|c|c|c|c|}
\hline \multirow{2}{*}{$\begin{array}{c}\text { Sample } \\
\text { code }\end{array}$} & \multirow{2}{*}{$\begin{array}{l}\text { Type of } \\
\text { Feldspar }\end{array}$} & \multirow[t]{2}{*}{ Provenance } & \multicolumn{3}{|c|}{ Composition on Unity } & \multirow{2}{*}{$\begin{array}{c}\mathrm{Fe} \\
(\mathrm{ng} / \mathrm{g})\end{array}$} \\
\hline & & & $\mathrm{KF}$ & $\mathrm{NaF}$ & $\mathrm{CaF}$ & \\
\hline R28 & $\mathrm{M}^{\#}$ & Switzerland & 0.95 & 0.05 & 0 & $\$$ \\
\hline R42 & $\mathrm{S}$ & China & 0.91 & 0.08 & 0.01 & 1185 \\
\hline R43 & $S$ & China & 0.91 & 0.08 & 0.01 & 943.2 \\
\hline R44 & M & Spain & 0.91 & 0.08 & 0.01 & 753.4 \\
\hline R47 & $S$ & China & 0.85 & 0.12 & 0.03 & 207.3 \\
\hline R49 & $S$ & China & 0.83 & 0.16 & 0.02 & 691.9 \\
\hline R50 & M & $\mathrm{u} / \mathrm{k}$ & 0.8 & 0.18 & 0.01 & 1597.4 \\
\hline R56 & M & $\mathbf{u} / \mathbf{k}$ & 0.52 & 0.46 & 0.02 & 2124.5 \\
\hline R57 & $\mathrm{M}$ & $\mathrm{u} / \mathrm{k}$ & 0.43 & 0.53 & 0.04 & 1537.3 \\
\hline
\end{tabular}




\begin{tabular}{lcccccc}
\hline R58 & $\mathrm{M}$ & $\mathrm{u} / \mathrm{k}$ & 0.27 & 0.63 & 0.1 & 1607.7 \\
R63 & $\mathrm{S}$ & Iceland & 0.03 & 0.29 & 0.69 & 36790.7 \\
R64 & $\mathrm{S}$ & Iceland & 0.01 & 0.15 & 0.85 & 11263.7 \\
\hline
\end{tabular}

Table 2 - PL decay lifetime data of $\mathrm{Fe}^{3+}$ measured using R56.

\begin{tabular}{llllll}
\hline Lifetime (ms) for non-resonant & $7 \mathrm{~K}$ & $103 \mathrm{~K}$ & $199 \mathrm{~K}$ & $295 \mathrm{~K}$ \\
$(4.77 \mathrm{eV})$ and resonant (3.29 & & & & \\
$\mathrm{eV}$ ) excitations & & & & & \\
$4.77 \mathrm{eV}$ & $\tau_{4.77}(\mathrm{~ms})$ & 5.07 & 4.43 & 3.79 & 2.97 \\
& & & & & and 0.90 \\
& & & & & \\
& $\tau_{3.3}(\mathrm{~ms})$ & 4.97 & 4.24 & 3.80 & 3.32 \\
$3.29 \mathrm{eV}$ & $\tau_{3.3} *(\mathrm{~ms})$ & 6.09 & 5.18 & 4.35 & 2.22 \\
\end{tabular}

\section{Highlights:}

- Excitation-energy dependent emission in $\mathrm{Fe}^{3+}$

- $\mathrm{Fe}^{3+}\left({ }^{4} \mathrm{~T}_{1} \rightarrow{ }^{6} \mathrm{~A}_{1}\right)$ relaxation kinetics dependent on excitation energy

- Dual origin of red-shifted $\mathrm{Fe}^{3+}$ emission at cryogenic temperatures

- Potential for site-selective mapping in heterogeneous feldspar 

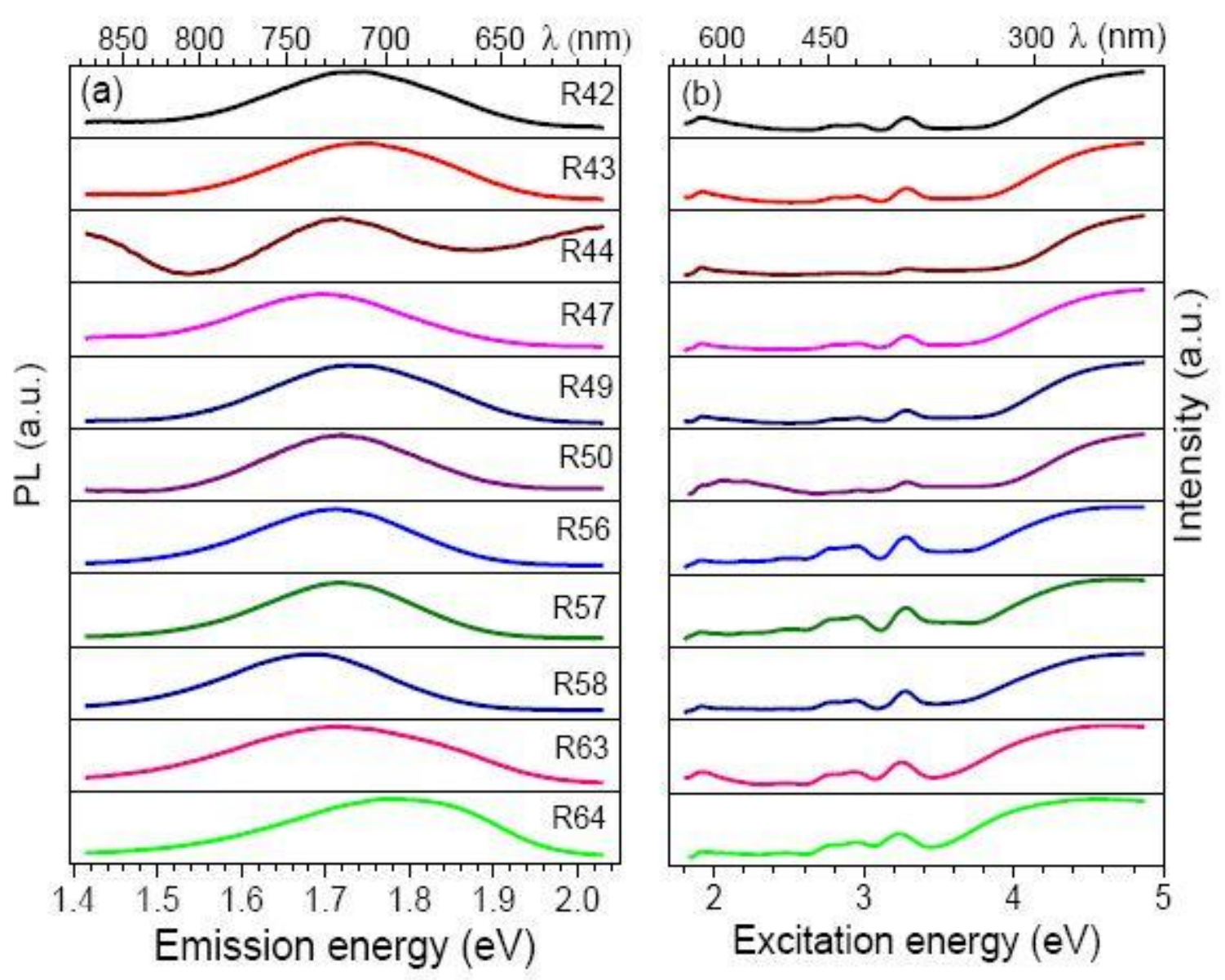


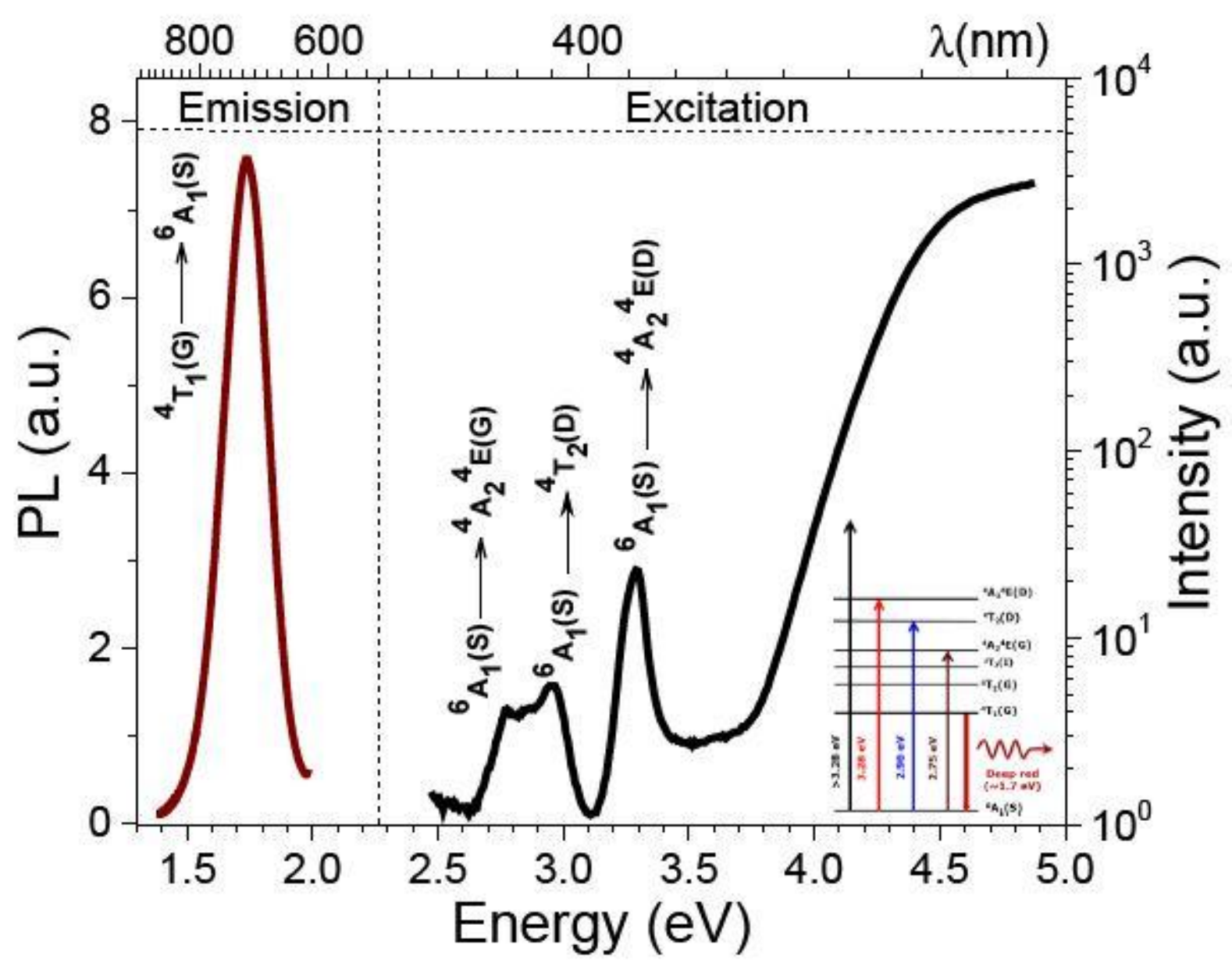



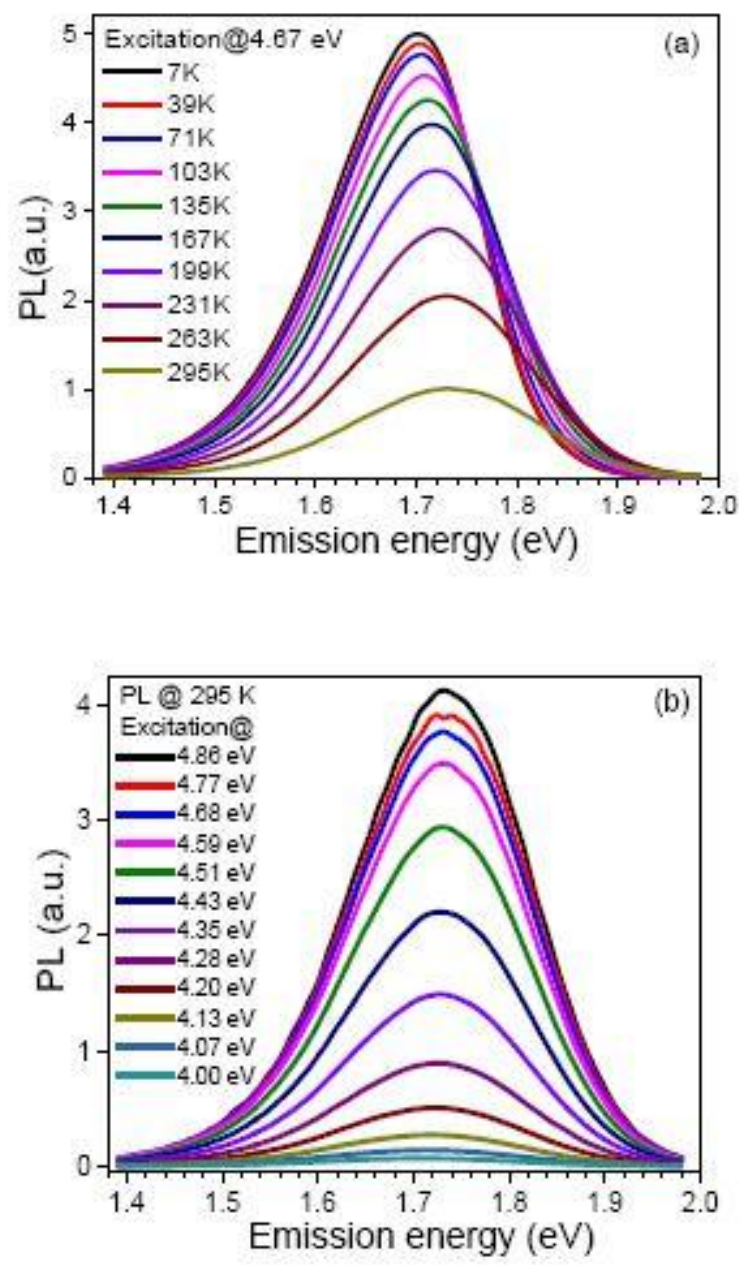

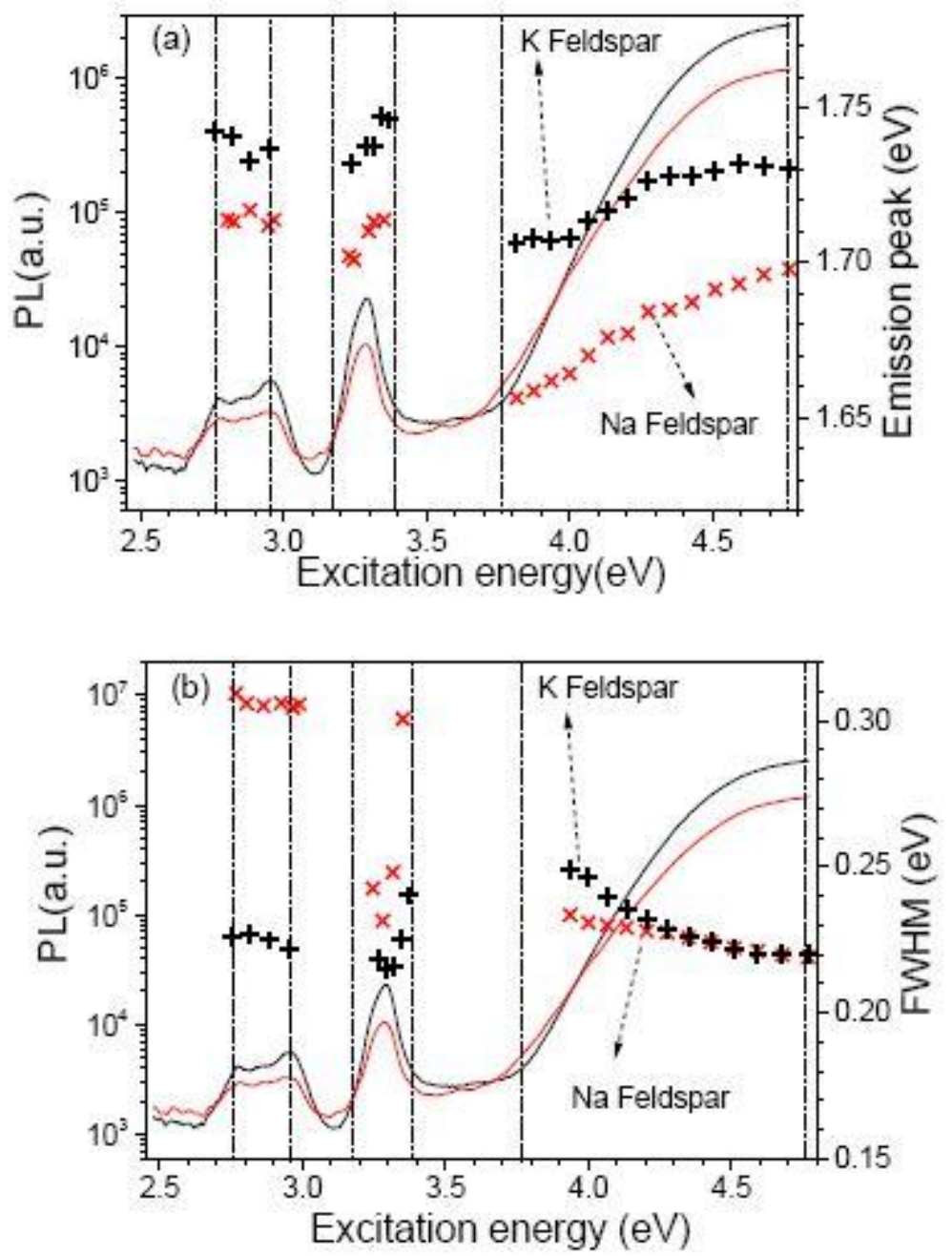

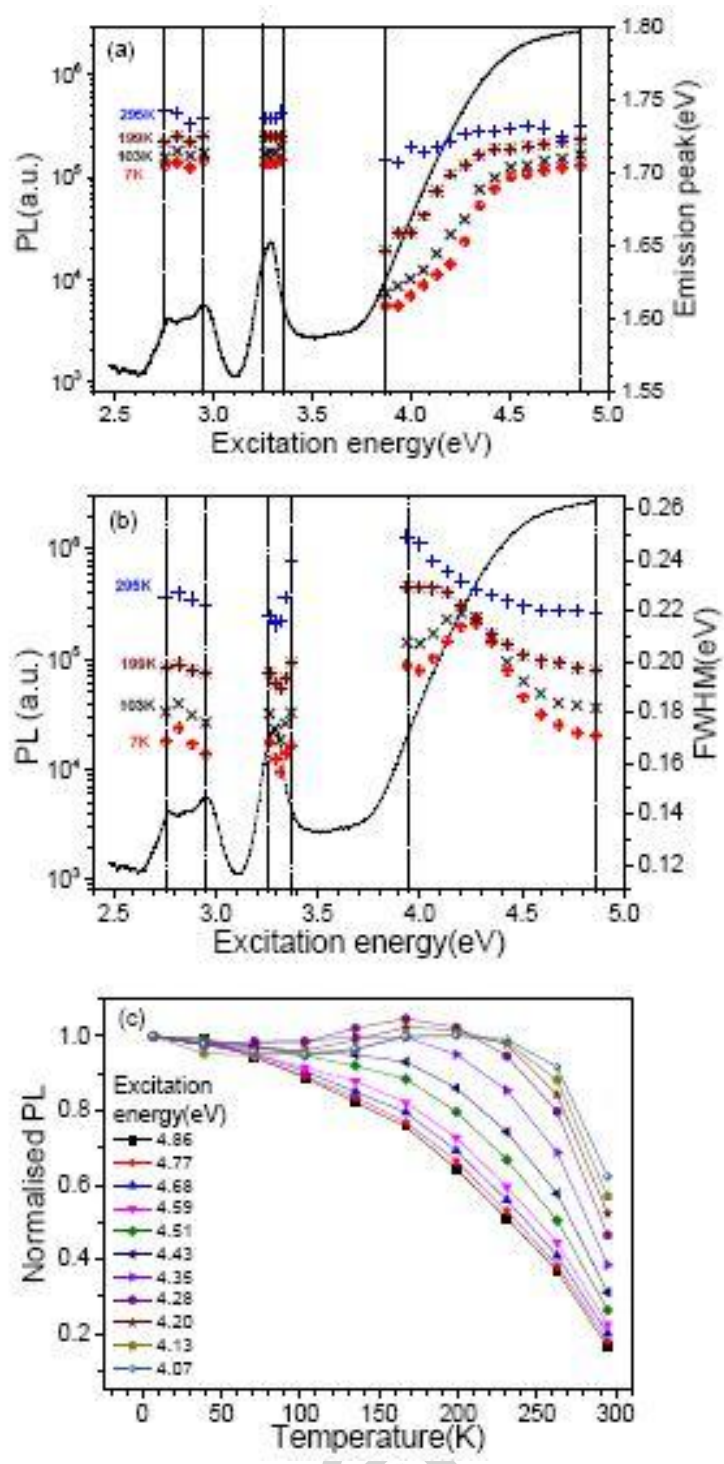

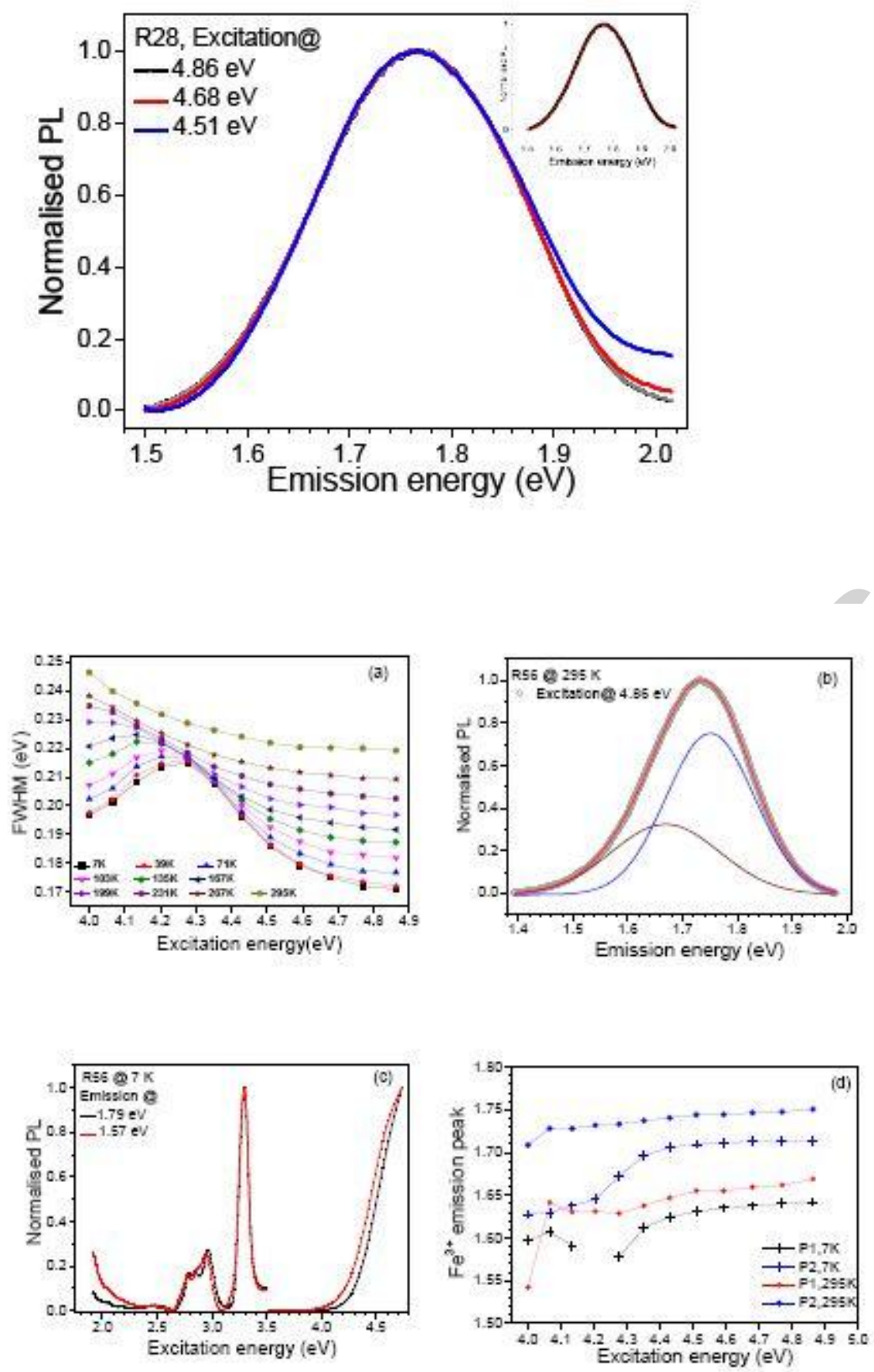

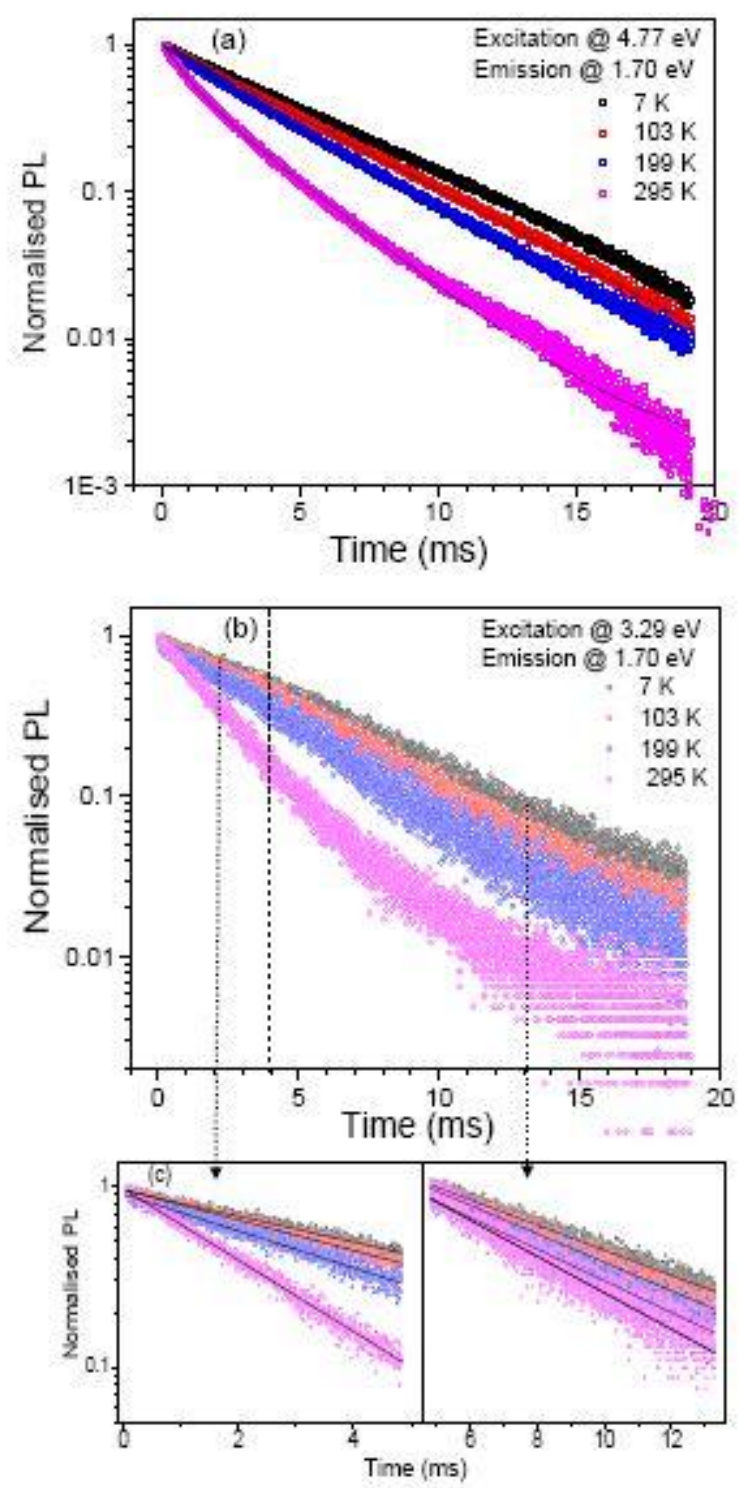


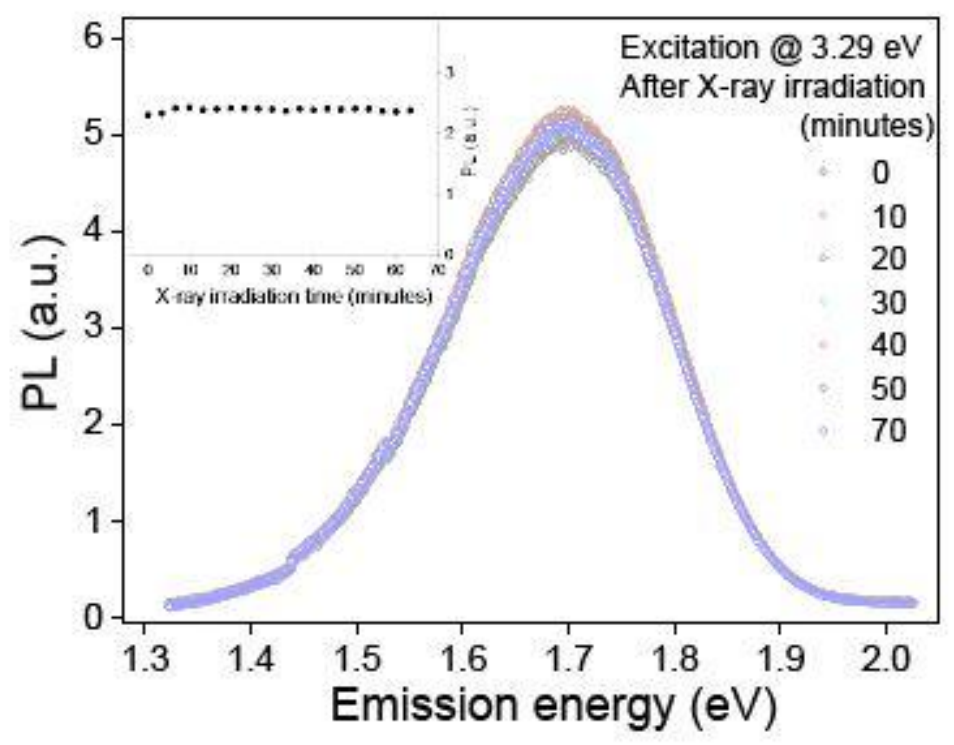

